\title{
PRELIMINARY EVALUATION OF THE ENDOVASCULAR TREATMENT OF INTRACRANIAL ANEURYSMS WITH DETACHABLE COILS IN VIGIL PATIENTS
}

\author{
José Roberto Falco Fonseca', Nitamar Abdala², Dárcio Roberto Nalli', \\ Marcos Hideki Idagawa33, João de Deus da Costa Alves Jr', \\ Márcio Chaves Pedro Marques ${ }^{3}$, Henrique Carrete Jr. ${ }^{1}$, \\ Roberto Gomes Nogueira ${ }^{4}$, Jacob Szjenfeld ${ }^{5}$
}

\begin{abstract}
Endovascular treatment of intracranial aneurysms with coil embolization became the most important therapeutic option with better morbidity and mortality rates and quality of life. Following immobility, patients are treated with general anaesthesia. Objective: To test viability of endovascular tre atment on wake patients. Method: Considering clinical symptoms, psychological characteristics and aneurysmal morphology, four patients with five intracranial aneurysms were selected. Results: Four among five cases were completed with this technique. Patient 1 was partially treated after 75 minutes presenting vesical stress. Patient 2 presented subarachnoid hemorrhage after aneurysmal re-rupture, and the procedure was completed under general anaesthesia. The other three patients presented no interc u rrences during the treatment. Conclusion: Endovascular treatment on wake patients with intracranial aneurysm can be an alternative to a selected group of patients.
\end{abstract}

KEY WORDS: intracranial aneurysm, embolization, endovascular treatment, anaesthesia, wakefulness patients.

\begin{abstract}
Avaliação preliminar do tratamento endovascular de aneurismas intracranianos com espirais destacáveis em pacientes no estado de vigília

RESUMO - O tratamento endovascular de aneurismas intracranianos com espirais destacáveis estabeleceuse como principal opção terapêutica na maioria dos pacientes, por menores taxas de morbidade e mortalidade. De forma geral, os pacientes são tratados sob anestesia geral, pela necessidade de imobilidade. Objetivo: Avaliar a viabilidade do tratamento endovascular de aneurismas intracranianos em pacientes no estado de vigília. Método: Cinco aneurismas foram tratados em quatro pacientes selecionados, considerando-se quadro clínico, a intenção de colaboração do paciente e características morfológicas do aneurisma. Resultados: A abordagem proposta foi possível em quatro dos cinco casos. O paciente 1 obteve compactação parcial do aneurisma, pois o tratamento foi interrompido por desconforto vesical. O paciente 2 a presentou hemorragia subaracnóidea por re-ruptura do aneurisma, e o tratamento foi completado no mesmo tempo sob anestesia geral. Nos demais casos, não houve intercorrências. Conclusão: 0 tratamento endovascular de aneurismas intracranianos em pacientes no estado de vigília é viável em grupo selecionado de pacientes.
\end{abstract}

PALAVRAS-CHAVE: aneurisma intracraniano, embolização, tratamento endovascular, anestesia, pacientes em alerta.

The endovascular treatment (EVT) of intracranial aneunsm (IA) with detachable coil (DC) has shown a meaningful growth world wise. This therapeutical modality has ceased to be an option to conventional surgery to become one of a choice. Several published articles in recent literature have pointed its advantages. Generally, they show better results when it comes to morbidity and mortality, less hospital admission time, faster recovery and less general cost for patients treated for ruptured aneurysms ${ }^{1-3}$ as well as for unruptured ones ${ }^{4,5}$. The basic start of this treatment consists in filling in the IA light with DC, focusing on a dense pack. This filling prevents from blood circulation inside the aneurysm protects it from a possible rupture. For the procedure to succeed, it is essential that the patient remains still. The immobil-

Diagnostic Imaging Department (DID), Federal University of São Paulo SP, Brazil: ${ }^{1} \mathrm{Hired}$ Doctor; ${ }^{2}$ P rofessor; ${ }^{3}$ Assistant Doctor; ${ }^{4}$ Professor and Vice-Chief; ${ }^{5}$ Professor and Chief.

Received 13 April 2006, received in final form 6 July 2006. Accepted 25 August 2006.

Dr. Roberto Gomes Nogueira - Rua Pará 126 / 52 - 01243-020 São Paulo SP - Brasil. 
ity is necessary for the digital mapping or roadmap ping, an angiographic tool utilized during the entire procedure to keep itself in perfect quality, allowing a safe microcatheterization and embolization. For this reason, literature in general recommends the patients to be treated under general anaesthesia. Therefore, analgesia is not something we look for on anaesthetic procedure Except for the anaesthetic blockade in the inguinal region on the initial stage of the treatment, the procedure is considered painless.

Currently, some research teams have opted in only treating patients under sedation ${ }^{6}$. The potential advantages on this option related to the general anaesthesia, besides avoiding endotracheal intubation and mechanic ventilation, includes less risk to the patient and a possible evaluation of the neurological state $^{6}$. However, sedation itself might bring some disadvantages. Since these medications interf e re in the conscious state of the patient, its use might harm the immediate neurological evaluation. Moreover, a paradoxical effect is described, a psychomotor agitation that some patients present when psychotropic drugs are given.

The appearance of such side effect, even though unusual, would not make the procedure possible. Thus, the authors of this study formulate the hypothesis that a cooperative patient with a favourable neurological state presenting a small or medium dimension intracranial aneurysm would be able to remain still during the critical moments of the procedure (macrocatheterization, microcatheterization and embolization), making the treatment possible on a wake state.

\section{METHOD}

Four patients with five intracranial aneurysms were selected to go under EVT on wake state in the period from January/2006 to March/2006. All of them had had a previous cerebral digital subtraction angiography. The main start for patients' inclusion was the predictability of a fast procedure which would not exceed 60-75 minutes, time considered comfortable for the patient. For that, the authors elected three general criteria which are (1) clinic state, (2) patients' intent to cooperate, and (3) operators' compliance to the standard endovascular technique treatment.

Inclusion criterion 1: clinic state - Patients without updated subarachnoid hemorrhage were selected. In other words, those who have an unruptured intracranial aneurysm and those who besides having an aneurysm rupture remained in a good neurological state (Hunt-Hess I) and found themselves in a late stage of evolution with over 14 days of ictus.

Inclusion criterion 2: selection of patient who intent to cooperate - In the eve of the treatment, patients were debriefed and it was explained to them details about the traditional EVT (with general anaesthesia) and this study's proposal. The patients considered cooperative were the ones who felt comfortable and whose perception was of a self advantage as well as beneficial, and the ones who were non-cooperative were the ones who showed themselves uncomfortable or apprehensive and favourable to traditional approach.

Inclusion criterion 3: operators' compliance to standard endovascular technique treatment - Standard endovascular technique was defined as the one which consists in direct microcatheterization and embolization of the aneurysm without the need of a containment system. For the operator's compliance to this technique, it was considered the following parameters: (1) aneurysm of up to $15 \mathrm{~mm}$ bigger in the axis and (2) ratio body / neck above 1.5. The ratio body / neck was defined as a relation between the biggest diameter of the aneurysm and the measure of the bosom.

The standard EVT consisted in an anaesthetic femoral blockage with Lidocaine $1 \%$, Seldinger, right femoris artery puncture, adapting the $6 \mathrm{~F}$ sheath, intravenous infusion of $10000 \mathrm{UI}$ of Heparin in pool and 6F catheter guide progression up to the cervical myotome of the inner carotid artery. We decided to name macrocatheterization the techniques used up to this stage. Following, the progress of the microcatheter-microguide joint through the catheter-guide and through the intracranial artery system up to the selective catheterization of the aneurysm (microcatheterization), deposition and the subsequent detachment of the DC (embolization), removal of the material from macro and microcatheterization and Heparin reversion with Protamine Sulfate.

Analyzed parameters:

Aneunsm features - Localization, state (unruptu red or ruptured), size (measure in millimeters of both bigger axis) and ratio body / neck.

Procedure features -Procedure time; success therapy rate; anatomic result; com plaints and interc u r rences during the proce du re; patient's clinical condition on hospital discharge.

The moment of the first acquisition of an angiography procedure was considered as the initial time (TO) and the moment of the last control angiogram as considered the final time (Tf). The time of the proce d u re (Tp) was established according to the formula: $\mathrm{Tp}=\mathrm{Tf}-\mathrm{TO}$. This inform ation was recovered minutes after the procedureand is available in the digital angiography equipment.

The successful therapy was defined as the procedure which can be finalized with the patient on wake state, independently of the final anatomic result. This result of the treatment was evaluated on the final control angiogram, being classified under total packing (no flow on IA), subtotal (small flow on the neck) and partial (flow on the neck and body) ${ }^{7}$.

The complaints and intercurrences were catalogued as they appeared.

The patient's clinic state on hospital discharge was classified as asymptomatic or as a neurological deficit bearer.

A professional anaesthesiologist followed the procedure with proper material and equipment in hand to apply 
Table 1. Patients clinical aspects and aneurysm morphology.

\begin{tabular}{cccccc}
\hline Case & Sex & Age (years) & State & Size $(\mathrm{mm})$ & Ratio Body / Neck \\
\hline 1 & M & 69 & ruptured & $15 \times 12$ & 3.7 \\
2 & F & 39 & ruptured & $5 \times 3$ & 2.5 \\
3 & F & 39 & unruptured & $4 \times 4$ & 2.0 \\
4 & F & 65 & ruptured & $7 \times 4$ & 2.3 \\
5 & M & 28 & unruptured & $5 \times 5$ & 2.5 \\
\hline
\end{tabular}

Table 2. Therapeutic results, intercurrences and complications in the five cases.

\begin{tabular}{|c|c|c|c|c|c|c|}
\hline Case & $\begin{array}{l}\mathrm{Tp} \\
(\mathrm{min})\end{array}$ & $\begin{array}{l}\text { Success } \\
\text { therapy }\end{array}$ & $\begin{array}{l}\text { Anatomic } \\
\text { result }\end{array}$ & Intercurrence & Complications & $\begin{array}{l}\text { Clinical condition at } \\
\text { hospital discharge }\end{array}$ \\
\hline 1 & 75 & Yes & Partial & Vesical stress & none & Asymptomatic \\
\hline 2 & 66 & No & Total & Re-rupture of aneurysm & SAH & Asymptomatic \\
\hline 3 & 45 & Yes & Total & $\begin{array}{l}\text { Headache during the coil } \\
\text { detaching }\end{array}$ & none & Asymptomatic \\
\hline 4 & 75 & Yes & Total & none & none & $\begin{array}{l}\text { Preexistent III nerve } \\
\text { palsy unaltered }\end{array}$ \\
\hline 5 & 48 & Yes & Total & none & none & Asymptomatic \\
\hline
\end{tabular}

$\mathrm{Tp}$, time of the procedure; $\mathrm{SAH}$, subarachnoid hemorrhage.

the general anaesthesia if necessary. All patients were treated on the Neurointervention and Digital Angiography Ward at the Diagnostic Imaging Department - UNIFESP. An angiography by Phillips, model V5000, with monitors and high resolution roadmapping was used.

The project was submitted to appreciation and was approved by the Ethics Council at UNIFESP. All patients who participated on the study read, understood, agreed and signed the Informed Consent Term.

\section{RESULTS}

The treatment was possible in four over five cases, providing a technical success rate of $80 \%$. The anatomic result was of total packing on three $(75 \%)$ and partial on one $(25 \%)$. The patient who had partial packing had undergone another treatment session out of this study protocol with a final result of subtotal packing. Four cases were accomplished following the proposed technique. One patient required general anaesthesia during the proceduredue to a hemorrhagic complication and had his treatment completed under general anaesthesia with an anatomic result of total packing. All other cases were treated within the estimated time limit. The complaints observed were vesical stress (case 1) and light and limited headache during the first detachable coil (case 3). Table 1 shows clinical information on the patients and IA treated features. Table 2 combined information of the procedures.
Evaluation of case 1 - Patient 1 presented vesical stress after 75 minutes from the beginning of the t reatment (T75). Even though cooperative, he started to move himself unintentionally, harming the next steps of the procedure, which was interrupted with the IA partial packing. Four days later, he had again undergone the treatment under general anaesthesia, reaching subtotal packing.

Evaluation of case 2 - At the time T33 during the microcatheterization, the patient complained about sudden holocranial headache, psychomotor agitation and depression on the conscience level. The clinical hypothesis of the re-rupture of the aneurysm was confirmed by angiographic control, which showed extravasations of the contrast medium from the aneurysm on (Fig 1). It was immediately induced general anaesthesia and the procedure was retaken (T41) and finalized (T66), reaching total aneurysm packing. This case was classified as a non-successful therapy because of the incapability to finalize it with the patient on wake state.

Evaluation of case 3 - After two weeks, case 2 patient was recovered. She was submitted to an EVT of the unru p t $u$ red IA of the superior right hypophyseal artery. She was asymptomatic throughout the procedure, except at the moment of the first detach- 


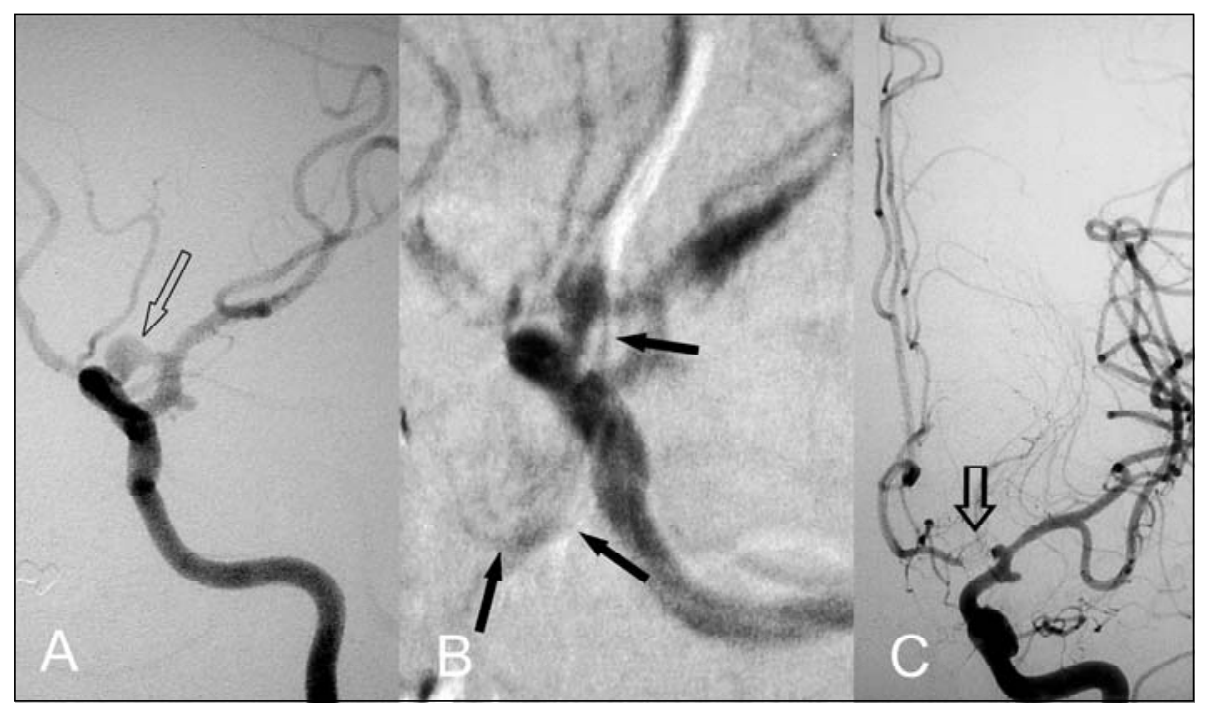

Fig 1. Endovascular treatment of an aneurism of the carotid bifur cation. (A) Angiogram of the inter nal left carotid artery, oblique inci dence. Note aneurism from its bi furcation (empty arrow). (B) Angio gram obtained at the moment of the rupture of the aneurism. The quality of the image is damaged due to a movement caused by the patient's restlessness. Observe in the point of rupture the overflow of the contrast medium (black ar rows). (C) Angiogram of final con trol. Note the total packing of the aneurism (large arrow).

Fig 2. Endovascular treatment of an aneurism in the upper hypophyseal artery. (A) Angiogram of the internal left carotid art e $r$, oblique incidence. Note the regular aneurism (black arrow). (B) An giogram of total control. Note the total packing of the aneurism (empty arrow).

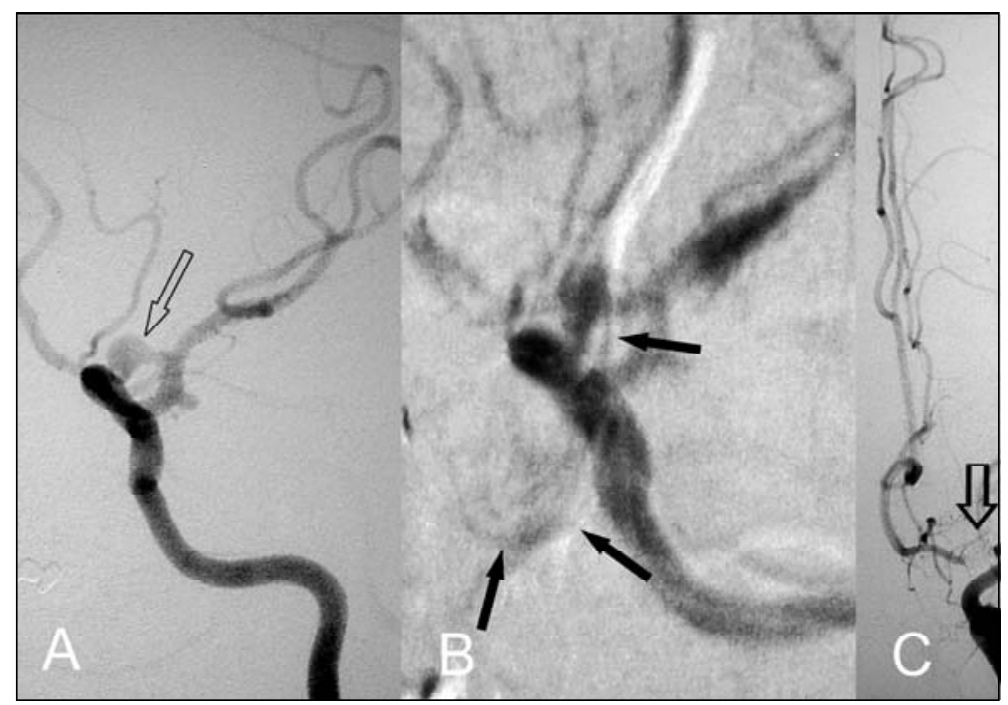

able coil, when she referred to a weak short-term headache. The painful illustration gave in spontaneously and there was no need for the treatment. The detachable coil mechanism was thermoelectric.

Evaluation of cases 4 and 5 - Patients 4 and 5 had their treatment completed at $\mathrm{T} 75$ e T48, respective$l y$, and had no complaints. Patient 4 hadpreexisting paralysis of the III cranial nerve. Patient 5 presented a superior hypophyseal artery aneurysm, same as case 3.

\section{DISCUSSION}

Kole et al. ${ }^{7}$ demonstrated an association among negative events on the follow up of embolized patients with an immediate angiographic result posttreatment. By negative events these authors consid- ered the increase of the volume of a remainder of the neck, the necessity of a retreatment, re-blood and death. These negative events were significantly associated to the non-total packing degree (partial or subtotal). Current literature, however, supports the idea that there is a protection to the new hemorrhages, even in cases of non-total packing ${ }^{2}$. In the p resent study, the anatomic result of total packing (75\%) could be considered satisfactory.

There were two complaints (urinary stress and headache during the detachable coil) without clinical consequences or severe intercurrence. Two patients showed no complaints throughout the procedure.

Four patients had asymptomatic hospital discharge $(80 \%)$, including the patient who suffered severe intercurrence. A patient was admitted to the hospi- 
tal due to a complete paralysis of the third left cranial nerve and his clinical picture remained stable until hospital discharge despite the treatment.

Some aspects should be considered in case 1. In these age ranges, the benign prostatic hyperplasia is $p$ revailing. The volume of liquid and of iodine contrast medium is meaningful. Therefore, the vesical drilling or external urinary collector must always be considered in patients with such profile. On the other hand, the dimensions of the intracranial aneurysm we re at the maximum recognizable limit $(15 \mathrm{~mm})$. If the patient had not presented discomfort, the Tp would have extrapolated the expected reading. It is possible that the best technique for this studied aneurysm would be to use the containment system.

Two important appreciations must be made about case 2 . First, since the patient was on wake state she could report her initial painful symptom and when she presented on the other typical symptoms and signs of $\mathrm{SAH}$, it helped to a faster diagnostic and consequent immediate therapeutical intervention on a complicated severe hemorrhage. This aspect may have been fundamental for the patient's good evolution. Under general anaesthesia, the diagnostic of such complicated hemorrhage can be delayed and it's usually done if there are only indirect clinical signs, such as increase of heart frequency and fluctuation of the arterial press u re. Second, the observed complication reinforces the need of an anaesthesiologist during the entire procedure of EVT of IA on wake patients ${ }^{8}$. The case 2 patient showed a ruptured aneurysm. A higher rate of complications related to endovascular treatment of ruptured aneurysm is mo re commonly re p o rted in medical literature than unruptured aneurysms ${ }^{9-11}$. However, Qureshi et al. ${ }^{6}$ did not observe such unprop ortional rate of higher complications in the group treated without general anaesthesia. In our study, it is considered that the appearance of the described complication has no relation at all to the endovascular technique applied.

In case 3, a low intensity electric current was applied on the outer extremity of the propeller system, which connected itself to the DC. It raises a potential diffe rence, electric current and heat emission and a little spot of the sound is broken, than occurring its detachment. The painful system was considered related to the IA localization. The aneurysms on the superior hypophyseal arte ry are located, limited and partially enclosed by a dura-mater recess known as carotid cave ${ }^{12}$. The electric current passage and the heat discharge in an innerved region such as the du- ra-mater must have been the cause of the symptom. It is suggested, there $f$ o re, using other discharge systems for the IA in this localization, such as pressure gradient. In the treatment of case 5, the DC was applied and its detachment process was done by the pressure gradient. The patient showed no symptoms. This fact corroborates the hypothesis given on case 3. Figure 2 exemplifies the EVT of IA using DC.

Few studies on the theme are available in medical literature. Qureshi et al. described results of 150 patients treated under sedation without general anaesthesia ${ }^{6}$. The procedurewas possible on 79 over 92 unruptured aneurysms (85.5\%) and in 53 over 58 ruptured aneurysms (91.3\%) with a rate of technical success of $88 \%$. The global complication index was of $7 \%$ for the group with unruptured aneurysm and of $12 \%$ for ruptured aneurysm patients. These figures did not differ significantly from other casuistryones evolving patients under general anaesthe$\mathrm{sia}^{9-11}$. These authors concluded that EVT of IA on woken patients can be safe and fiseable. However, they did not choose patients considering the IA size and morphology, the endovascular technique used and probable time of the procedure. The non-successful therapy rate obtained was mainly due to the unfavourable morphological features of the IA which demanded more complex and long-lasting endovascular technique. In the present study, patient selection was limited and it reached a $75 \%$ technical success rate. Different from Qureshi et al. study ${ }^{6}$, there was no sedation and the patients were treated on wake state. This aspect enabled a better clinic control over the patients. Another used restriction in this study was the operator's enrollment to the standard endovascular technique aiming to reduce the $\mathrm{Tp}$. This technique demands an aneurysm bigger than 1.5 from the ratio body/neck. However, there are available endovascular technique for IA treatment with a large neck and unfavourable morphology, such as those using a definitive temporary containment system with balloons ${ }^{13}$ with neurostent, presenting good results ${ }^{14,15}$. It was chosen not to go through these techniques since they're considered long-lasting and more complex. Though, currently, newthree-dimensional DC has been enabling EVT of a part of an IA of large neck without using the containment system presenting good results ${ }^{16,17}$. Finally, the joint usage of new technology has been pointed as responsible for the late good endovascular results ${ }^{18}$.

There are scattered reports of major surgical treatments in other departments without endotracheal 
intubation and mechanic ventilation ${ }^{19,20}$, although the authors did not recover in medical literature related studies on EVT of IA on patients on wake state, up to the present date.

In conclusion, although the few amount of studied cases, EVT of IA on patients on wake state have shown to be viable when the patients are properly chosen.

\section{REFERENCES}

1. Van der Shaaf I, Algra A, Wermer M, et al. Endovascular coiling versus neurosurgical clipping for patients with aneurysmal subarachnoid haemorrhage (Cochcrane Review). In: The Cochcrane Library, Issue 1, 2006; Oxford: Update Software.

2. Molyneux A, Kerr RSC, Ly-Mee Y, et al. International subarachnoid aneurysm trial (ISAT) of neurosurgical clipping versus endovascular coiling in 2143 patients with ruptured intracranial aneurysm: a randomised comparison of effects on survival, dependency, seizures, rebleeding, subgroups, and aneurysm occlusion. Lancet 2005;366:809-817.

3. Gallas S, Pasco A, Cottier JP. A multicenter study of 705 ruptured intracranial aneurysms treated with Guglielmi detachable coils. Am J Neuroradiol 2005;26:1723-1731.

4. Wiebers DO, Whisnant JP, Huston $\mathrm{J} 3^{\text {rd }}$, et al. Unruptured intracranial aneurysms: natural history, clinical outcome, and risks of surgical and endovascular treatment. Lancet 2003;362:3-10.

5. Lee T, Baytion M, Sciacca R, Mohr JP, Pile-Spellman J. Aggregate analysis of the literature for unruptured intracranial aneurysm treatment. Am J Neuroradiol 2005;26:1902-1908.

6. Qureshi AI, Suri MF, Khan J, et al. Endovascular treatment of intracranial aneurysm by using Guglielmi detachable coils in awake patients: safety and feasibility. J Neurosurg 2001;94:880-885.

7. Kole MK, Pelz DM, Kalapos P, Lee DH, Gulka IB, Lownie SP. Endovascular detachable coil of intracranial aneurysms: important factors related to rates and outcomes of incomplete occlusion. J Neurosurg 2005;102:607-615.
8. Luginbuhl M, Remonda L. Interventional neuroradiology: recent developments and anaesthesiologic aspects. Minerva Anestesiol 1999;65:445454.

9. Niskanen M, Koivisto T, Rinne J. Complications and postoperative care in patients undergoing treatment for unuru ptu redintracranial aneurysm. J Neurosurg Anestesiol 2005;17:100-105.

10. Ross IB, Dhillon GS. Complications of endovascular treatment of cerebral aneurysms. Surg Neurol 2005;64:12-19.

11. Park HK, Horowitz M, Jungreis C et al. Periprocedural morbidity and mortality associated with endovascular treatment of intracranial aneurysms. Am J Neuroradiol 2005;26:506-514.

12. Alleyne CH, Coscarella E, Spetzler RF, Walker MT, Patel AC, Wallace RC. Microsurgical anatomy of the clinoidal segment of the internal carotid artery, carotid cave, and paraclinoid space. Barrow Quarterly 2002;18:8-10.

13. Moret J, Cognard C, Weill A, et al. The "remodeling" technique in the treatment of wide neck intracranial aneurysms. Interv Neuroradiol 1994;3:21-35.

14. Lee YJ, Kim DJ, Suh SH, Lee SK, Kim J, Dim DI. Stent-assisted coil embolisation of intracranial wide-necked aneurysms. Neuroradiology 2005;47:680-689.

15. Buenos Aires experience with the Neuroform self-expanding stent for the treatment of intracranial aneurysms. J Neuro su rg2005;102:235-241.

16. Vallée JN, Pierot L, Bonafé A, et al. Endovascular treatment of widenecked aneurysms using three-dimensional coils: predictors of immediate anatomic and clinical results. Am J Neuroradiol 2004;25:298-306.

17. Vallée JN, Pierot L, Mont' alverne F, et al. Unuruptu red intracra nial aneurysms treated by three-dimensional coil embolisation: evaluation of postoperative aneurysm occlusion volume. Neuroradiology 2005;47:438-445.

18. Katz JM, Tsiouris AJ, Biondi A, et al. Advances in endovascular aneurysm treatment: are we making a difference? Neuroradiology 2005;47: 695-701.

19. McGregor MC, Koler AJ, Labat GC, Perni V, Hirko MK, Rubin JR. Awake aortic aneurysm repair in patients with severe pulmonary disease. Am J Surg 1999;178:121-124.

20. Luders JC, Steinmetz MP, Mayberg MR. Awake craniotomy for microsu rgical obliteration of mycotic aneurysms: technical report of three cases. Neurosurgery 2005;56(Suppl 1):S201. 